\title{
Consideraciones anestésicas en el síndrome de HELLP
}

\author{
Hellp syndrome and anesthesia
}

Florencia Galarraga1, Ana Schwartzmann²

\begin{abstract}
The HELLP syndrome is one of the most relevant obstetrical pathologies but for the anestesiologist, which, can even condition the anesthetic technique before a caesarean section. The early colocation of a epidural catheter in obstetrical pathologies that can finish in a cesarean section with the aim of diminishing the mortality associated to the pathology is still under discussion. We present a case of a 25 years old patient programmed for a caesarean section, with probable diagnosis of HELLP syndrome, In which a epidural catheter was placed 12 hours before the surgery presenting a trombocitopenia of $86,000 / \mathrm{mm}^{3}$. Our objetive is to discuss the issues of the anesthetic management in patients with HELLP syndrome and the advantages of the early placement of a epidural catheter.
\end{abstract}

\section{RESUMEN}

El síndrome de HELLP es una de las patologías obstétricas más relevantes para el anestesiólogo, la cual, puede incluso condicionar la técnica anestésica ante una eventual cesárea. Actualmente, está en discusión la colocación de un catéter epidural temprano en patologías obstétricas que puedan terminar en una cesárea, con el objetivo de disminuir la morbimortalidad asociada a ella. Presentamos el caso de una paciente de 25 años programada para una operación cesárea, con probable diagnóstico de síndrome HELLP a la que se le colocó un catéter epidural 12 horas antes de la cirugía con una trombocitopenia de $86 \times 10^{3} /$ uL. Se presenta con el objetivo de discutir las directivas del manejo anestésico de las pacientes con síndrome de HELLP y las ventajas de la colocación temprana de un catéter epidural.

\section{Key words:}

HELLP syndrome, platelet count, anesthesia peridural

\section{Palabras clave:}

Síndrome de HELLP, recuento de plaquetas, anestesia peridural

\footnotetext{
Residente de la Cátedra de Anestesiología y Reanimación. Asistente del Departamento de Farmacología.

2 Profesora Adjunta de la Cátedra de Anestesiología y Reanimación (UdeLaR). Experta en medicina del dolor y cuidados paliativos.
}

Fecha de recepción: 21 de junio de 2018

Fecha de aceptación: 31 de julio de 2018

\section{ORCID}

https://orcid.org/0000-0001-5687-8208

\section{Correspondencia:}

Ana Schwartzmann

E-mail: alfincito@gmail.com 


\section{Introducción}

$\mathrm{E}$ I síndrome de HELLP forma parte de las complicaciones severas de la preeclampsia, su nombre surge del acrónimo en inglés, de las alteraciones que lo definen: hemólisis, elevación de las enzimas hepáticas y plaquetopenia[1],[2],[3].

Se desarrolla en $0,1 \%$ a $0,8 \%$ del total de los embarazos y en el $10 \%$ a $20 \%$ de los embarazos con preeclampsia severa o eclampsia. Es una de las formas más graves dentro de los estados hipertensivos del embarazo, aunque entre $15 \%$ y $20 \%$ de las pacientes no presentan hipertensión o proteinuria previamente. Presenta una mortalidad materna del $1 \%$ a $24 \%$ y una mortalidad fetal entre $7 \%$ a $60 \%[1],[4]$.

Hay cuatro tipos de trastornos hipertensivos relacionados con el embarazo: preeclampsia, hipertensión crónica, hipertensión gestacional y preeclampsia sobreagregada a hipertensión gestacional. El diagnóstico de preeclampsia se realiza por cifras de presión arterial mayores a 140/90 $\mathrm{mmHg}$ en dos to$\operatorname{mas}[1],[2],[3],[4],[6]$.

El diagnóstico del síndrome de HELLP se realiza con: Plaquetas menores a $100.000 / \mathrm{mm}^{3}$, aumento de transaminasas hepáticas, glutámico oxalacético (TGO) mayor de $40 \mathrm{UI} / \mathrm{l}$ o dos veces su valor normal y glutámico pirúvico (TGP) mayor de 70 UI/l. Anemia hemolítica microangiopática con presencia de esquistocitos. Elementos de hemólisis como aumento de la bilirrubina indirecta mayor o igual a $1,2 \mathrm{mg} / \mathrm{dl}$, incremento de la lactato deshidrogenasa (LDH) mayor a $600 \mathrm{UI} / \mathrm{l}$ o más de dos veces su valor normal[1],[3].

Los factores de riesgo para síndrome de HELLP incluyen: multiparidad, edad materna mayor a 25 años, raza blanca, antecedentes personales de aborto, diabetes mellitus, hipertensión arterial crónica, enfermedad renal y obesidad[1],[3],[5].

Con plaquetas menores a $50.000 / \mathrm{mm}^{3}$ o fibrinógeno menor a $300 \mathrm{mg} / \mathrm{dl}$ se considera en proceso de coagulación intravascular diseminada (CID), agravando el pronóstico.

Las técnicas anestésicas regionales son de elección, presentando una mortalidad materna reducida, menor pérdida sanguínea y mejor analgesia postoperatoria. No existen diferencias entre las técnicas neuroxiales, aunque habría una ventaja teórica de la anestesia epidural por la menor incidencia de hipotensión grave[6].

El riesgo de hematoma epidural asociado con la anestesia regional se relaciona con los trastornos de la coagulación más que con la trombocitopenia[7]. Sin embargo, en pacientes portadoras de síndrome HELLP con recuento de plaquetas en descenso es recomen- dable la colocación de un catéter peridural cuando aún las plaquetas estén en rango de seguridad[6],[7].

\section{Caso clínico}

Paciente de 25 años, trasplantada renal, programada para operación cesárea cursando 26 semanas de gestación por síndrome de HELPP.

Se realiza diagnóstico de síndrome de HELLP, por presentar: plaquetas en descenso y hemólisis leve. Múltiples cirugías con anestesia general sin incidentes.

Cursando embarazo bien controlado, Anemia crónica, sin síndrome hemorragíparo. No epigastralgia, no cefalea.

Con planteo de síndrome de HELLP se decide interrupción del embarazo, luego de completada la maduración pulmonar fetal.

Se decide colocación de catéter epirural (CPD) precoz, con plaquetas en rango de seguridad, y realización de anestesia peridural para la cesárea, previa obtención de consentimiento informado. Se coloca CPD con valores de trombocitopenia de $86 \times 10^{3} / \mathrm{uL}$.

Doce horas previo a la cirugía. La intervención quirúrgica se realiza con anestesia peridural sin incidentes en base a bupivacaína al 0,5\% y fentanyl 100 gammas por CPD. Se realiza monitorización estándar de la American Society of Anesthesiology, hidratación en base a solución Ringer con lactato a $1 \mathrm{ml} / \mathrm{kg} / \mathrm{h}$.

En el intraoperatorio se continúa infusión de sulfato de magnesio que venía recibiendo desde el diagnóstico a $1 \mathrm{~g}$ cada 6 horas.

Acto quirúrgico sin incidentes, recién nacido con puntaje en la escala de Apgar 6/9, se ventila con máscara facial con oxígeno.

Postoperatorio en área monitorizada por 24 horas, se mantiene infusión de sulfato de magnesio. Se continua el tratamiento con dexametasona $10 \mathrm{mg}$ iv cada 12 horas.

Se retira CPD a las 30 horas de realizada la cirugía con recuento plaquetario de $101.000 / \mathrm{mm}^{3}$, sin incidentes.

\section{Discusión}

La anestesia regional es considerada ampliamente como la técnica de elección para la realización de una cesárea en pacientes portadoras de síndrome HELLP con el fin de evitar complicaciones como la hemorragia intraventricular o aquellas secundarias a la aspiración broncopulmonar. La presencia de coagulopatía 


\begin{tabular}{|c|c|c|c|c|c|}
\hline \multicolumn{6}{|c|}{ Exámenes de laboratorio en orden cronológico } \\
\hline & $19 / 1-6: 34$ & 19/1 - 17:00 & $20 / 1-11: 00$ & $20 / 1-15: 14$ & $21 / 1-22: 14$ \\
\hline Hemoglobina g/dl & 9,0 & 8,5 & 10,9 & 9,7 & 7,6 \\
\hline Plaquetas $/ \mathrm{mm}^{3}$ & 103.000 & 86.000 & 57.000 & 93.000 & 101.000 \\
\hline $\begin{array}{l}\text { Tiempo de pro- } \\
\text { trombina }\end{array}$ & $85 \%$ & $73 \%$ & $68,3 \%$ & $96 \%$ & $100 \%$ \\
\hline KPTT seg & 34 & & 32,5 & & 29 \\
\hline TGO/TGP U/L & $23 / 7$ & & $22 / 8$ & $27 / 9$ & \\
\hline $\mathrm{BT} / \mathrm{Bl} \mathrm{mg} / \mathrm{dl}$ & $0,37 / 0,16$ & & $0,29 / 0,12$ & $0,45 / 0,18$ & \\
\hline $\mathrm{LDH} \cup / \mathrm{L}$ & 815 & & & & \\
\hline $\begin{array}{l}\text { Fosfatasa alcalina } \\
\text { U/L }\end{array}$ & 41 & & 23 & 46 & \\
\hline Fibrinógeno & & & 329 & 464 & \\
\hline
\end{tabular}

clínicamente significativa, es una contraindicación absoluta para el uso de esta técnica[6].

En cuanto al recuento plaquetario, la tendencia suele ser más importante que el número absoluto de plaquetas, no habiendo un valor mínimo aceptable que se haya identificado como aquel por encima del cual se puedan realizar técnicas neuroaxiales con seguridad[6].

Las recomendaciones actuales incluyen no realizar anestesia neuroaxial en pacientes con plaquetas menores a $50.000 / \mathrm{mm}^{3}$. Con valores entre 50.000 y $100.000 / \mathrm{mm}^{3}$ plaquetas, se deben obtener pruebas adicionales de la coagulación previo a la realización de una anestesia neuroaxial, obteniendo valores normales, es segura la realización de una anestesia regional[5].

Actualmente, los valores de plaquetas utilizados responden a una variedad de mediciones, que incluyen mediciones de la amplitud del tromboelastograma en pacientes sanas y con preeclampsia severa con plaquetas menores a $100.000 / \mathrm{mm}^{3}$. No existen diferencias entre la técnica regional a utilizar y la seguridad entre la técnica epidural o raquídea no está estudiada. En ausencia de otras anormalidades de la coagulación, el riesgo de hematoma asociado con anestesia neuroaxial, con plaquetas mayores a 75.000 es muy bajo[8].

Los corticoides están indicados ya que independientemente del efecto fetal, se ha demostrado que éstos a altas dosis (dexametasona $10 \mathrm{mg}$ iv. cada 12 horas), mejoran el número de plaquetas, disminuyen los niveles de enzimas hepáticas y prolongan la latencia entre el diagnóstico y el parto de forma dosis dependiente, se deben mantener en el posparto para evitar un efecto rebote[9].
Dentro del manejo anestésico en estas pacientes, en la valoración paraclínica, se debe incluir: hemograma, crasis sanguínea y función renal, además, se pueden solicitar pruebas viscoelásticas de la coagulación y ecocardiograma, como parte de la valoración de la preclampsia.

- Hemograma: el límite de plaquetas actualmente aceptado para realizar una técnica regional es de $75.000-80.000 / \mathrm{mm}^{3}$, con valores mayores no hubo reportes de hematomas espinales.

- Crasis sanguínea: en pacientes con factores de riesgo para CID, como HELLP o desprendimiento prematuro de placenta normoinserta se debe solicitar precozmente la crasis.

- Pruebas viscoelásticas: no permiten predecir el riesgo de hematoma espinal, luego de la realización de una técnica regional.

La importancia de la analgesia del parto en éstas pacientes está dada por la reducción de las respuestas hipertensivas mediadas por el dolor. Además, la presencia de un catéter peridural funcionante permite la eventual realización de una cesárea[10].

\section{Conclusión}

Al enfrentarse a una paciente con síndrome de HELLP con recuentos plaquetarios en descenso, las recomendaciones son:

- Recuentos plaquetarios por encima de 100.000/ $\mathrm{mm}^{3}$ son seguros para la realización de anestesia neuroaxial.

- Recuentos entre 50.000 y $100.000 / \mathrm{mm}^{3}$ en pacientes sin evidencia clínica ni paraclínica de coa- 
gulopatía (equimosis, petequias, gingivorragia, epíxtasis o sangrados en los sitios de venopunción), es segura la realización de técnicas regionales.

- Recuentos menores de 50.000 plaquetas requieren la transfusión de concentrados plaquetarios para la realización de una cesárea, independientemente de la técnica anestésica empleada[10],[11].

Negar la realización de una anestesia epidural a una paciente a causa de un valor arbitrario de plaquetas no sería lo más adecuado, especialmente en pacientes con preeclampsia severa. La hipertensión arterial y la taquicardia secundarias a la intubación orotraqueal puede desencadenar una hemorragia cerebral, siendo ésta, una de las principales causas de mortalidad en madres con preeclampsia[6].

La analgesia del parto es el método ideal para el alivio del dolor en estas pacientes, permitiendo además, la continuación a anestesia para una eventual cesárea[9].

Concluimos que la colocación de un CPD precoz cuando el recuento plaquetario viene descendiendo en el contexto de un síndrome HELLP, aún en pacientes que no estén en trabajo de parto, nos asegura poder ofrecerle a la paciente la técnica que ha demostrado mayores beneficios para el binomio materno fetal a la hora de realizarse una operación cesárea.

\section{Referencias}

1. Vigil-De Gracia P, Silva S, Montufar C, Carrol I, De Los Rios S. Anesthesia in pregnant women with HELLP syndrome. International Journal of Gynecology \& Obstetrics [Internet]. Wiley; 2001 Jun 22;74(1):23-7.

2. Shirozu K, Kuramoto S, Kido S, Hayamizu K, Karashima Y, Hoka S. Hematoma After Transversus Abdominis Plane Block in a Patient With HELLP Syndrome. A \& A Case Reports [Internet]. Ovid Technologies (Wolters Kluwer Health); 2017 May;8(10):257-60.

3. Mammaro A, Carrara S, Cavaliere $A$, Ermito $S$, Dinatale $A$, Pappalardo EM, et al. Hypertensive disorders of pregnancy. J Prenat Med. 2009 Jan;3(1):1-5.

4. American College of Obstetricians and Gynecologist. issuing body II, Title: Hypertension, pregnancy-induced practice guideline. WQ 244.

5. Crosby E, Preston R. Obstetrical anesthesia for a parturient with preeclampsia, HELLP syndrome and acute cortical blindness. CAN J ANAESTH 1998/45: 5/pp 452-459.

6. Dennis A. Management of preeclampsia: issues for anaesthetists. Anesthesia 2012. The association of anaesthetists of Great Britain and Ireland 2012, 67, 1009-1020.

7. Martínez A, Cano M, Palacios A, Mateo P. Manejo Anestésico del Síndrome Hellp. Revista Colombiana de Anestesiología, Sociedad Colombiana de Anestesiología y Reanimación, Bogotá, Colombia vol. XXXI, núm. 1, 2003.

8. Zuccolotto E, Pagnussatt E, Nogueira G, et al. Anesthesia in pregnant women with HELLP syndrome: case report. Centro de Ensino e Treinamento em
Anestesiologia (CET-SBA) da Clínica de Anestesiologia de Ribeirão Preto (CARP), Ribeirão Preto, SP, Brazil; Available online 1 October 2016.

9. Basaran A, Basaran M, Basaran B, Sen C, Martin JN. Controversial clinical practices for patients with preeclampsia or HELLP syndrome: a survey. J Perinat Med. 2015 Jan;43(1):61-6.

10. Palit $S$, Palit $G$, Vercauteren $M$, Jacquemyn $Y$. Regional anaesthesia for primary caesarean section in patients with preterm HELLP syndrome: a review of 102 cases. Clin Exp Obstet Gynecol. 2009;36(4):230-4.

11. Rueda J, Pinzón C, Vasco M. Manejo anestésico para operación cesárea urgente: revisión sistemática de la literatura de técnicas anestésicas para cesárea urgente. Revista colombiana de Anestesiología. 2012; 40 (4): 273-286. 